\title{
A Hairpin Antenna-in-Package Concept for RFID Tag Applications
}

\author{
T. Papatheologou, A.B. Smolders, and U. Johannsen \\ Eindhoven University of Technology, Department of Electrical Engineering, Den Dolech 2, \\ 5600 MB Eindhoven, The Netherlands \\ Phone: (+31) 040-2474356, Email: T.Papatheologou@tue.nl
}

\begin{abstract}
Antenna integration is a relative new concept for the miniaturization of Radio Frequency (RF) transceivers where a key problem is to achieve a small size at low cost. Traditional solutions, using ceramic materials, are too costly. Additionally, the current trend in RFID applications is the development of smaller and cheaper tags. In this paper, we present an elegant and low-cost Antenna-in-Package (AiP) solution for RFID tag applications. A printed dipole antenna that integrates the balun function with improved filtering characteristics is designed on a low-cost dielectric material. Simulation and measurement results verify the performance of the proposed antenna.
\end{abstract}

Index Terms - Antenna-in-Package (AiP), antenna integration, balun, Radio Frequency Identification (RFID) tags.

\section{INTRODUCTION}

Radio Frequency Identification (RFID) technology has become a mainstream application used in several fields such as: public transport, ticketing, access control, animal identification, and industrial automation [1]. RFID technology enables the identification and tracking of a distant object using radio waves. Moreover, it can incorporate more data, or even measure ambient conditions, such as temperature [2]. These possibilities reveal the importance of introducing this technology in the logistic chains of perishable goods (i.e., fruit, flower, medicine). Therefore, RFID technology could greatly improve the efficiency during shipment and guarantee the product quality when compared to the existing logistic chains that result in large amounts of waste. However, the mass introduction has not been realized yet due to the high-cost solutions that exist today.

Perishable mAnagement through Smart Tracking of lifEtime and qUality by RFID (PASTEUR) is the topic of a European project that involves the collaboration of universities and industries from the Netherlands, Belgium, Spain, and Austria. The goal of the project is the investigation and realization of an RFID-based sensor platform. The platform will track ambient conditions in order to guarantee product quality and establish a more efficient quality control in the logistic chains of perishable goods. The main technological drivers are the miniaturization of electronic systems together with lowcost and energy efficient system integration. Part of this work is also the investigation and integration of the RFID tag antenna for the development of highly integrated RF transceivers.

Moving to the $5.8 \mathrm{GHz}$ band has enabled the design of smaller RFID antenna structures. Moreover, the development of system-in-package ( $\mathrm{SiP})$ modules has offered the possibility to integrate a complete system with the necessary passive components into a single packaging module [3]. However, the antenna size is comparable to the wavelength of operation $(\sim 52 \mathrm{~mm}$ in air at $5.8 \mathrm{GHz})$ and the antenna is usually external to the integrated circuit (IC). Therefore, in order to achieve a higher degree of integration, a relative new, elegant solution in antenna design, the Antenna-in-Package (AiP), has been investigated. The AiP technology integrates an antenna or multiple antennas in the chip package [4], saving board area and cost, and maximizing the performance of a RF module. Several antenna concepts have been proposed in the literature. In [5]-[8] microstrip patch antennas and Printed Inverted-F Antennas (PIFA) are realized on ceramic materials, resulting in thin packages. The low temperature co-fired ceramic (LTCC) substrate technology offers high dielectric constants and an arbitrary number of layers for the miniaturization of microwave and millimeter wave circuits [9]. However, it is a costly solution, preventing the mass production of the antenna designs.

In this paper, we present an elegant and low-cost AiP solution operating around $6 \mathrm{GHz}$. A printed dipole is realized on top of a ground plane on a thin, low-cost material. The proposed concept can be used for realizing plastic packages using molding techniques. Moreover, the antenna solution integrates the balun function and achieves a sharp resonance with improved filtering characteristics, which can be beneficial for certain RFID applications. Finally, the proposed antenna has a significant smaller size as compared to the structure reported in literature [4].

\section{ANTENNA DESIGN}

Previous AiP studies mostly used antenna concepts based on the microstrip patch antenna and the PIFA. Common to both designs is that they require a ground plane. The presence of the ground plane is of vital importance in AiP designs since it serves as the shielding mechanism that protects the active circuitry from the 
antenna radiation. For the case of a microstrip line antenna the presence of a ground plane in a very close distance results in a high quality factor. Consequently, the line behaves as a microstrip resonator with very low radiation losses. Zhang et al. designed a microstrip line $\mathrm{AiP}$ in LTCC technology for single-chip RF transceivers in the $5.8 \mathrm{GHz}$ frequency band [4]. In this study, they proposed an easily configurable structure for adjusting the resonance frequency, in close proximity to the ground plane. The antenna achieved high radiation efficiency by the appropriate geometrical configuration of the line. The achievements in [4] with respect to AiP designs served as inspiration for our design. However, we consider the use of LTCC substrate technology as a drawback since it increases the cost of the final solution.

The goal of this study is to realize a smaller and cheaper AiP solution, which is compatible with plastic packaging technologies. Therefore, as a first step in our approach, we transferred the design of [4] onto a dielectric material (RO3003) with a dielectric constant of 3 [10]. An AiP with a board area equal to $25 \times 25 \mathrm{~mm}^{2}$ and a simulated radiation efficiency of $73.3 \%$ was achieved. Fig. 1 shows a photograph and the measured input impedance of the antenna. Hence, the realization of an AiP on substrates of lower permittivity is proven. However, the lower $\varepsilon_{r}$ has resulted in a larger element size.

In order to reduce the size of the antenna solution, the design of a half-wavelength printed dipole is investigated.

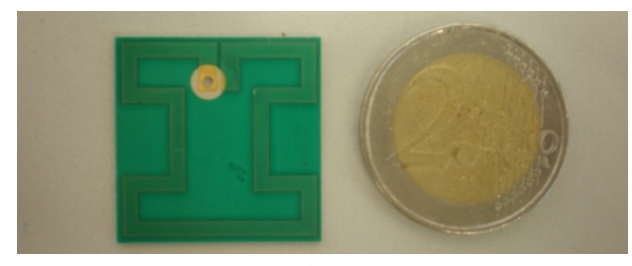

(a)

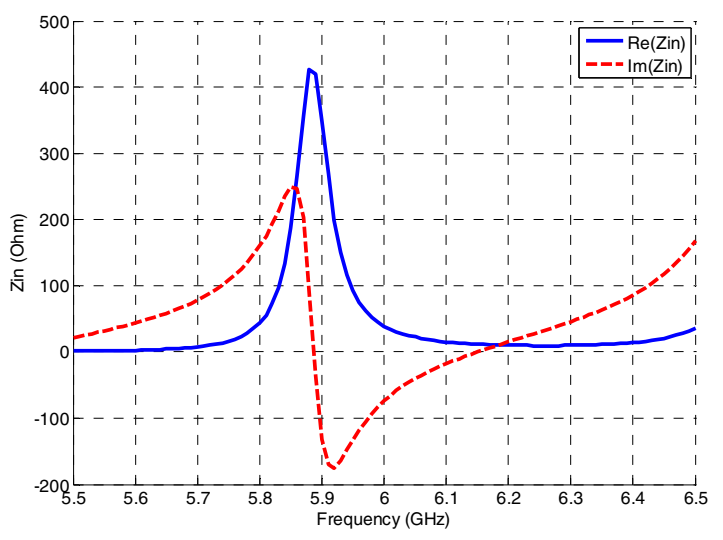

(b)

Fig. 1. (a) Photograph of the manufactured microstrip line antenna and (b) the measured input impedance.
The distance from the ground plane affects the dipole's resonant resistance. The input impedance and, thus, the radiation resistance of a dipole requires a specific value for matching purposes. For thin substrates the radiation resistance of the first resonance is very small since the dipole's first resonance is of series-type and the power that is radiated is also small [11]. In addition, dipoles are differential structures. Targeting a single-ended chip, it is necessary to connect a balun to the antenna. This will result in additional space requirements, more losses, and would increase the production yield time and assembly cost. In order to overcome these inherent problems, a new feeding structure is designed. The structure integrates the balun function and results in a higher resonant resistance that can be matched to the input impedance of the IC at the desired frequency. It is based on parallel-coupled, halfwavelength resonator hairpin-line filters [12] and improves the frequency selectivity of the antenna. Fig. 2 shows the top and side views of the proposed AiP. As shown, it is composed of three microstrip lines that are closely coupled. The third line is a half-wavelength dipole for operation at $6 \mathrm{GHz}$. The substrate material used is NelcoNX9 with a dielectric constant of 3 [13]. The resonance frequency of the antenna can be easily adjusted by changing the length $1_{4}$ of the dipole (see Fig. 2).

The commercial software HFSS was used for optimizing the antenna [14]. The design parameters are summarized in Table I. The resulting board area is $9.58 \mathrm{x}$ $17.8 \mathrm{~mm}^{2}$ for operation at $6 \mathrm{GHz}$. Furthermore, the substrate height is selected to be $\mathrm{h}=0.5 \mathrm{~mm}$ in order to prevent the excitation of the $\mathrm{TE}_{1}$ surface wave mode [11], and for the total volume of the structure to remain small. The simulated resonance frequency is $5.991 \mathrm{GHz}$ and the impedance bandwidth for which $\mathrm{S} 11<-10 \mathrm{~dB}$ is $38 \mathrm{MHz}$ from $5.972 \mathrm{GHz}$ to $6.01 \mathrm{GHz}$. A simulated radiation efficiency and gain of $61.5 \%$ and $3.27 \mathrm{dBi}$, respectively,

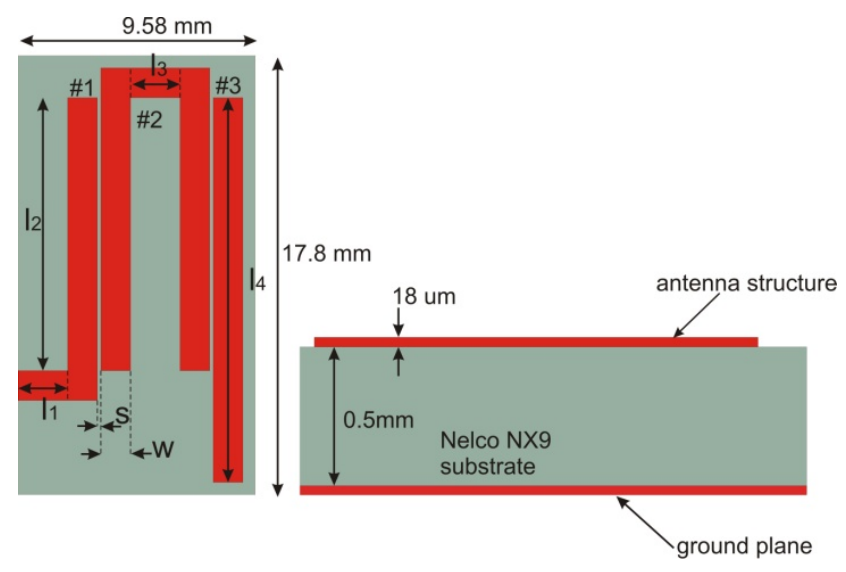

Fig. 2. Top and side views of the hairpin antenna. 
TABLE I

ANTENNA DESIGN PARAMETERS

\begin{tabular}{|c|c|}
\hline Parameters & Values (in mm) \\
\hline $\mathrm{I}_{1}$ & 2 \\
\hline $\mathrm{I}_{2}$ & 11 \\
\hline $\mathrm{I}_{3}$ & 2 \\
\hline $\mathrm{I}_{4}$ & 15.6 \\
\hline $\mathrm{W}$ & 1.2 \\
\hline S & 0.14 \\
\hline
\end{tabular}

are achieved. In Fig. 3 the magnitude of the current density on the structure is depicted.

Finally, Fig. 4 shows our proposed antenna integration concept in a ball grid array (BGA) package that carries the RF chip. Three dielectric layers, two buried, and a top metal layer are depicted (see also [5]). The radiating element is realized in the top metal layer. The bottom buried metal layer is used for the chip base and the signal traces, while the upper buried layer provides the antenna ground plane. The antenna is excited with a via through a circular aperture in the ground, which connects to the chip with bondwires and traces. Finally, external solder balls connect to the chip through bond wires, signal traces and vias.

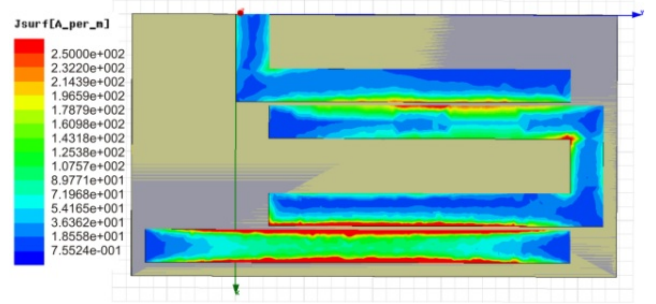

Fig. 3. Magnitude of current density of the hairpin antenna.

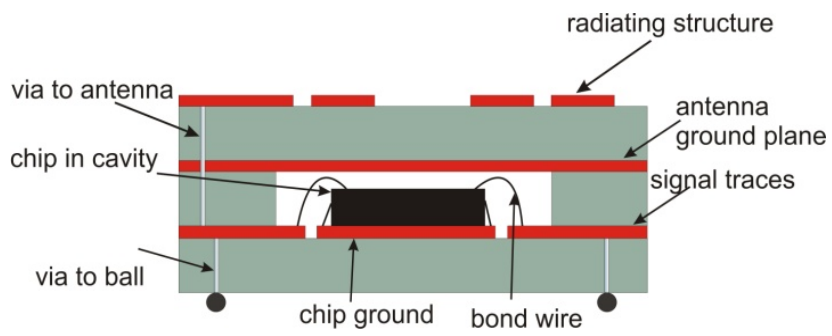

Fig. 4. Side view of the antenna integrated in a BGA package.

\section{MEASUREMENT RESULTS}

The proposed AiP was fabricated in Nelco NX9. A photograph of the manufactured prototype with the attached connector is shown in Fig. 5. In order to allow space for the connector, the feed line was extended $\left(l_{1}=6\right.$ $\mathrm{mm})$. For comparison purposes, simulations that included the extra line length and a model of the connector were performed in HFSS [14]. In Fig. 6, both measurement and simulation results of the input impedance are shown. Fig. 7 depicts the simulated and measured S11. A frequency shift of $88 \mathrm{MHz}$ can be observed. Additionally, the measured imaginary part gives higher values. This is considered to be the result of the connector since a simplified simulation model has been analyzed. Also,

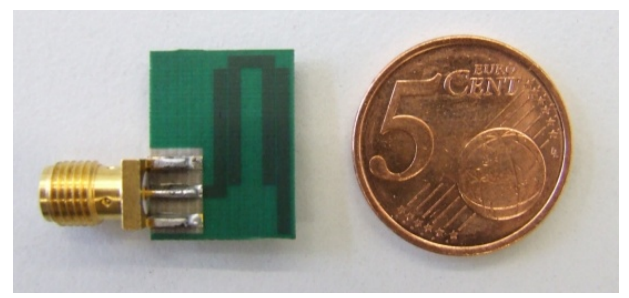

Fig. 5. Photograph of manufactured hairpin AiP.

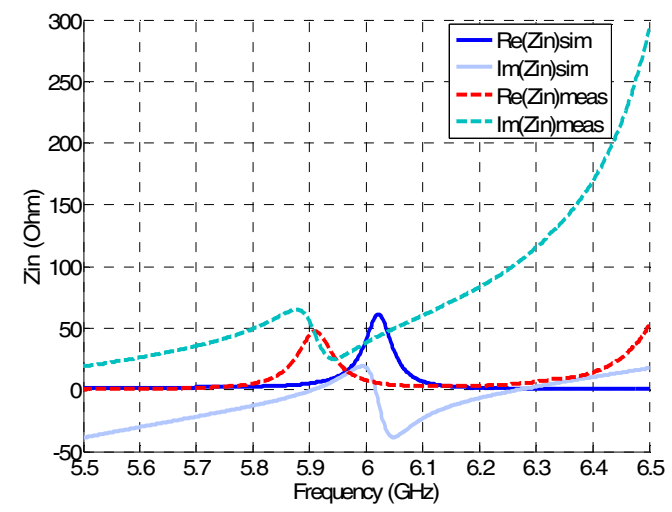

Fig. 6. Simulated and measured input impedance of the hairpin AiP.

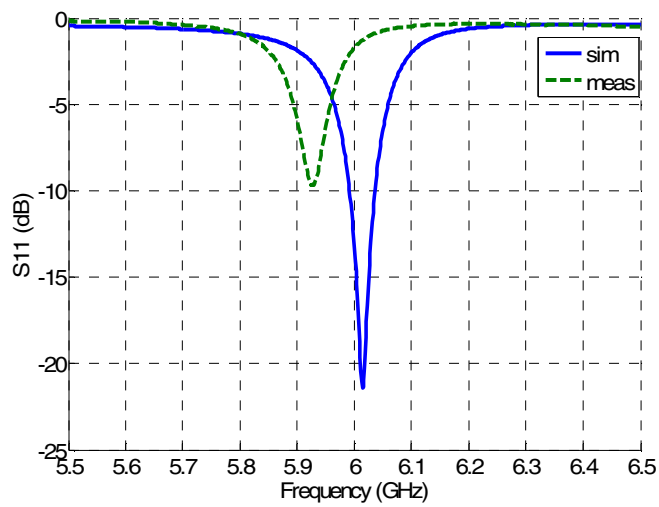

Fig. 7. Simulated and measured S11 of the hairpin AiP. 
manufacturing and permittivity tolerances can result in a lower resonance frequency [13]. Finally, the higher inductive imaginary part results in a deteriorated S11. The measured resonance frequency is $5.926 \mathrm{GHz}$ and the bandwidth for which $\mathrm{S} 11<-6 \mathrm{~dB}$ is $50 \mathrm{MHz}$ while the simulated bandwidth is $80 \mathrm{MHz}$ for resonance at 6.014 $\mathrm{GHz}$.

The radiation patterns for the two principal planes at $5.93 \mathrm{GHz}$ are shown in Fig. 8. The measurements were performed in an anechoic chamber and a close agreement with the simulations was found. The resulted radiation pattern has a maximum radiation towards $0^{\circ}$ in the E-plane and towards $22^{\circ}$ in the H-plane. This is considered to be the result of the printed dipole being at the edge of the ground plane. A low backside radiation is also achieved, serving the purpose of effectively shielding the die from the antenna radiation. The low signal level in the H-plane around $270^{\circ}$ is the result of the connector that blocks the signal path. Also, the differences of the signal level in the lower hemisphere of the E-plane can be seen as the result of the signal strength being close to the noise floor. Finally, the measured peak gain in the H-plane is equal to $2.84 \mathrm{dBi}$.

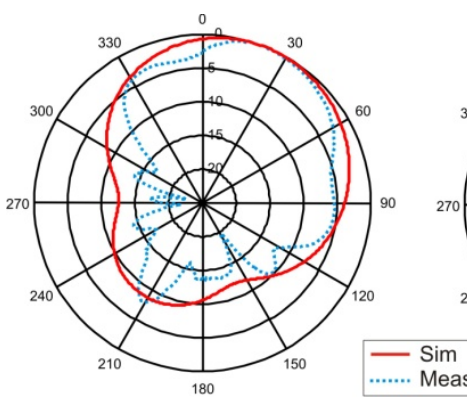

(a)

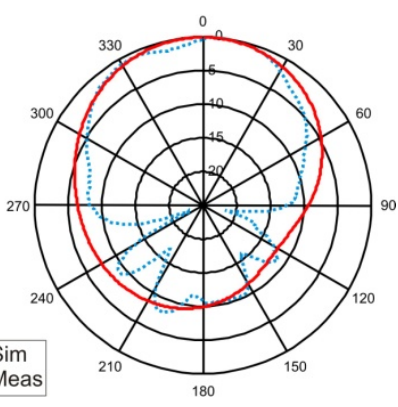

(b)
Fig. 8. Normalized radiation patterns of the manufactured hairpin antenna: (a) $\varphi=0^{\circ}$ (H-plane), and (b) $\varphi=90^{\circ}$ (E-plane).

\section{CONCLUSION}

In this paper a small, low-cost AiP design for RFID applications around $6 \mathrm{GHz}$ was presented. A dipole antenna with an integrated balun function and improved filtering characteristics was designed on a thin, low-cost material with low dielectric constant. Finally, the performance of the antenna was measured and verified.

The antenna achieved good operation around $6 \mathrm{GHz}$ while providing a single-ended solution with improved filtering characteristics that is beneficial for RFID applications. Additionally, it is a low cost antenna that can be integrated with plastic packages resulting in a reduced size and cost of the final product.

\section{ACKNOWLEDGEMENT}

The authors wish to thank the members of the European Catrene project PASTEUR for funding this research.

\section{REFERENCES}

[1] K. Finkenzeller, RFID Handbook: Fundamentals and Applications in Contactless Smart Cards and Identification, $2^{\text {nd }}$ ed., New York: J. Wiley \& Sons, March 2003.

[2] R. Want, "An introduction to RFID technology," Pervasive Computing, IEEE , vol. 5, no. 1, pp. 25-33, Jan.-March 2006.

[3] A. B. Smolders, N. J. Pulsford, P. Philippe, F. E. van Straten, "RF SiP: the next wave for wireless system integration," Radio Frequency Integrated Circuits (RFIC) Symposium, 2004. Digest of Papers. 2004 IEEE, vol., no., pp. 233-236, 6-8 June 2004.

[4] Y. P. Zhang, M. Sun, W. Lin, "Novel Antenna-in-Package design in LTCC for single-chip RF transceivers," Antennas and Propagation, IEEE Transactions on, vol. 56, no. 7, pp. 2079-2088, July 2008.

[5] Y. P. Zhang, "Integration of microstrip patch antenna on ceramic ball grid array package," Electronics Letters, vol. 38, no. 5, pp. 207-208, 28 Feb 2002.

[6] Y. P. Zhang, "Integrated circuit ceramic ball grid array package antenna," Antennas and Propagation, IEEE Transactions on, vol. 52, no. 10, pp. 2538-2544, Oct. 2004.

[7] S.-H. Wi, Y.-B. Sun, I.-S. Song, S.-H. Choa, I.-S. Koh, Y.S. Lee, J.-G. Yook, "Package-level integrated antennas based on LTCC technology," Antennas and Propagation, IEEE Transactions on, vol. 54, no. 8, pp.2190-2197, Aug. 2006.

[8] Y. P. Zhang, W. B. Li, "Integration of a planar inverted F antenna on a cavity-down ceramic ball grid array package," Antennas and Propagation Society International Symposium, 2002 IEEE, vol.4, no., pp.520-523, 2002.

[9] I. Wolff, "Design and technology of microwave and millimeterwave LTCC circuits and systems," Signals, Systems and Electronics, 2007, ISSSE '07. International Symposium on , vol., no., pp.505-512, July 30 2007-Aug. 2 2007.

[10] "RO3000 Series High Frequency Circuit Materials," Rogers Corporation, Data Sheet, www.rogerscorp.com.

[11] R. Garg, P. Bhartia, I. Bahl, and A. Ittipiboon, Microstrip Antenna Design Handbook, London: Artech House, 2001.

[12] R. Levy, R. V. Snyder, G. Matthaei, "Design of microwave filters," Microwave Theory and Techniques, IEEE Transactions on, vol. 50, no. 3, pp.783-793, Mar 2002.

[13] "NELCO N9000, PTFE Laminates", Park electrochemical Corporation, Data Sheet, www.parkelectro.com.

[14] http://www.ansoft.com/products/hf/hfss/ 